\title{
Using TMY3 data to stochastically predict the performance of a PV/VRB microgrid
}

\author{
K. M. Speidel* , A. C. Elmore, J. D. Guggenberger \\ Missouri University of Science and Technology, 1870 Miner Circle, Rolla, MO 65409, United States
}

\begin{abstract}
Accurately characterizing the performance of an off-grid photovoltaic (PV) microgrid system can help ensure that the system is appropriately sized to reduce the reliance on supplemental power generation via diesel-fueled generators. However, deterministic models cannot account for the inherent variability of solar insolation, ambient temperature, initial battery charge, and electrical load. A Monte Carlo model was developed by identifying those four variables as random variables. Typical Meteorological Year 3 (TMY3) data were used to develop the Probability Density Functions (PDFs) for the environmental variables, and the initial charge PDF was developed using engineering judgment while the load PDF was based on observed data. Comparison of the stochastic model results against limited performance data from two PV-based microgrid systems with vanadium redox batteries in Missouri indicated that the stochastic technique has the potential for widespread applicability. This potential is due in part because TMY3 datasets are available throughout the United States, and the basic model may be modified to include energy storage systems other than the subject vanadium redox battery.
\end{abstract}

Keywords: Microgrid, photovoltaic, Monte Carlo, stochastic

\section{Introduction}

Electrical microgrids are systems that can be used to increase the efficiency of power delivery to neighborhoods or areas that are typically served by utility companies as discussed by Provata et al. [1] and Patterson et al. [2], or they are systems that are used to provide power to locations that do not have access to utility -provided power such as those described by Merei et al. [3] and Bandara et al. [4]. This paper addresses the second category of microgrids, specifically those that use a combination of renewable energy sources such as photovoltaic (PV) to produce power, back-up diesel-powered generators, and batteries to store energy. Unlike the utility-connected first type, the second type of microgrid must balance power demand (load) with the available power supplies. Fossati et al. [5] describe how the design of such a microgrid balances the capital costs associated with the PV component and the battery component with the operating costs associated with a fossil-fuel generator for an expected electrical load profile. Performance prediction models are used to estimate the operational frequency of the fossil-fuel generator considering the environmental conditions such as solar insolation and ambient temperature that are variables in the production of PV power. Those environmental factors may also impact the operational efficiency of the energy storage devices. For example, Vanadium Redox Batteries (VRBs) and other batteries are impacted by ambient temperature [6].

Several models have been developed to model these systems. Alenezi et al. [7] developed an iterative approach to sizing a grid-connected PV system and found that technical outputs such as the number of PV modules, PV panel configuration, and inverter sizing are most important of the algorithm for integration to the grid. Huang et al. [8] found that the performance of a PV system is mainly influenced by weather,

\footnotetext{
* Manuscript received April 21, 2015; revised September 16, 2015.

Corresponding author. Tel.: +0-001-573-341-6784; E-mail address: kms5y2@mst.edu.

doi: $10.12720 /$ sgce.4.4.336-345
} 
solar irradiance, and module temperature and were able to develop an evolutionary programming algorithm to predict the performance of a grid-connected PV system based on those influences. Guggenberger et al. [6] created a microgrid performance model which characterized the performance of a PV/VRB microgrid based on the operation of a backup diesel generator. This deterministic model used typical meteorological year 3 (TMY3) data for the Global Horizontal Irradiance (GHI) and temperature input values for a microgrid located at Fort Leonard Wood in Pulaski County, Missouri. The model produced reasonable results, but it did not account for the inherent randomness in the environmental and load variables used in the calculations.

Arabali et al. [9] describe the development of a stochastic model that uses Monte Carlo Simulation (MCS) methods to simulate a PV and wind-turbine powered system with energy storage to supply a deferrable Heating Ventilation and Air Conditioning (HVAC) load. Their work used case studies to show the usefulness of their approach at the electric utility service scale. Others including Kishore and Fernandez [10] and Khatod et al. [11] have used MCS to simulate the performance of PV and wind-based microgrids, but those efforts have typically assumed traditional lead-acid or similar energy storage components.

This paper describes the development of a MCS model based on the Guggenberger et al. [6] deterministic model for a PV/VRB microgrid. The model includes four random variables: GHI, ambient temperature, VRB state of charge (SOC), and energy load. The stochastic analysis was performed for two microgrid systems, called TA-246 and B2222, located at Fort Leonard Wood. The probability density functions for GHI and ambient temperature were developed from typical meteorological year 3 (TMY3) data for that location. Three different initial VRB SOC conditions were evaluated: operating the microgrid for one year with a single randomly generated initial SOC at day one; operating the microgrid for one year with a unique initial SOC randomly generated at the beginning of each month, and operating the microgrid for one year where the initial SOC was charged to the upper threshold percent at the beginning of each month. These scenarios were used to evaluate three different system commissioning strategies: the system operated automatically for a year; the system operated automatically for single months; and the VRB was manually recharged at the beginning of each month.

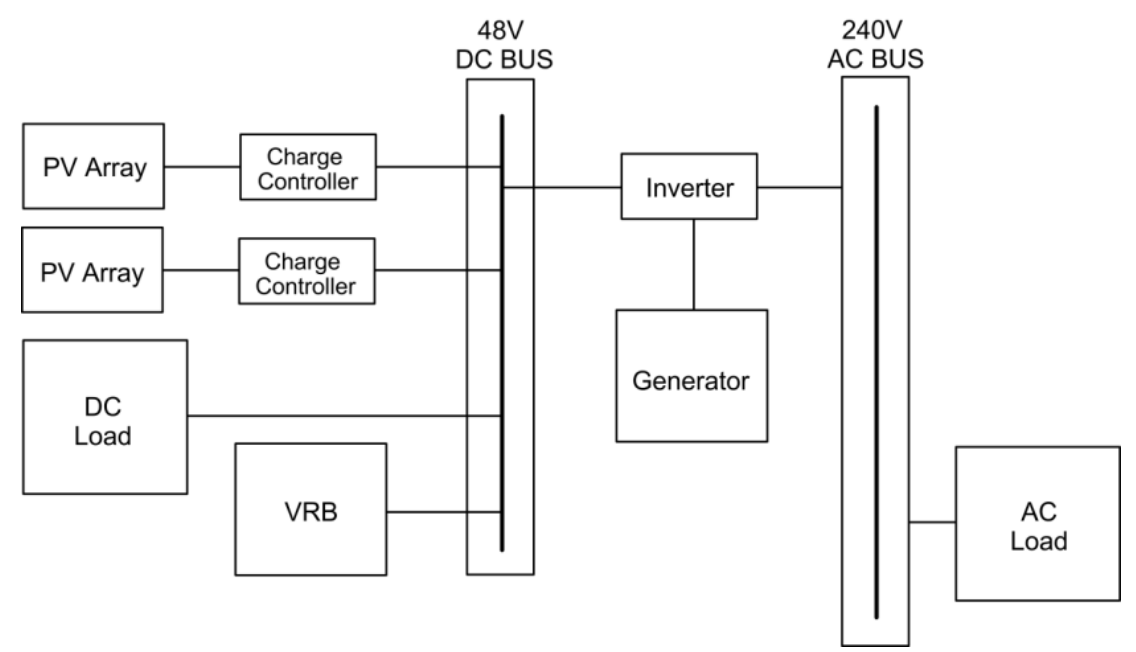

Fig, 1. Microgrid layout.

\section{Microgrid Descriptions}

\subsection{Microgrid TA-246}

Microgrid TA-246 was constructed at an off-grid Forward Operating Base (FOB) training area at Fort Leonard Wood, Missouri. The primary purpose of an FOB microgrid is to avoid the use of multiple, 
independent generators that are not optimally matched to their corresponding loads. The location latitude is 37.71 degrees and longitude is $-\mathbf{9 2 . 1 5}$ degrees. The following system description is summarized from Guggenberger et al. [6]. The off-grid microgrid consisted of a $6 \mathrm{~kW}$ PV array that included $30-200 \mathrm{~W}$ Brightwatts Inc. solar panels (model BI-156-200W-G27V). The PV system was separated into two $3 \mathrm{~kW}$ arrays and was mounted with a fixed horizontal angle of 38 degrees facing due south. The panels were connected to two Outback FlexMax 80 charge controllers that were used to charge a nominal 38-cell Prudent Energy $5 \mathrm{~kW}$ VRB with a rated energy density of $20 \mathrm{kWh}$. A three-cylinder Kubota diesel engine was connected to a Leroy Somer $8 \mathrm{~kW}$ brushless self-regulated generator. The generator was connected to the VRB using a Xantrex DC/AC inverter charge controller, and the system was controlled to minimize the use of the diesel-powered generator. Fig. 1 shows a schematic of microgrid TA-246. High-power density ultracapacitors were temporarily tested to supplement the VRB during intermittent periods of high power demand; however, this type of load shifting was not central to the experimental goals of the research and the use of the ultracapacitors was terminated.

\subsection{Microgrid B2222}

Microgrid B2222 was constructed at a site at Fort Leonard Wood, MO at latitude 37.71 degrees and longitude -92.15 degrees. The following system description is summarized from Nguyen et al. [12]. The microgrid consisted of a $15 \mathrm{~kW}$ PV array which included 54 - $280 \mathrm{~W}$ Suntech solar panels (model STP280-24/Vd). The system was separated into three $5 \mathrm{~kW}$ arrays and was mounted with a fixed horizontal angle of 38 degrees facing due south. The array was connected to three Outback FlexMax 80 charge controllers which were used to charge a 38-cell Prudent Energy 5kW VRB with a rated energy density of $20 \mathrm{kWh}$. The system supplied power to a nearby building load when the microgrid (PV and/or VRB) had sufficient power to satisfy the load. If insufficient renewable resources were available, a transfer switch was flipped and the building operated on the utility grid. The VRB was only charged by the PV arrays, but the deterministic and stochastic analyses presented in this paper were performed by assuming that that microgrid included the previously described diesel generator instead of the grid connection.

\section{Methods}

The random variables used in the model included GHI, ambient temperature, initial VRB SOC, and energy load. The U.S. Department of Energy's National Renewable Energy Laboratory (NREL) maintains the National Solar Radiation Database (NSRDB) which is a ready source for hourly data including irradiance, ambient temperature, and wind speed for over 1,000 locations across the United States. Wilcox and Marion [13] state that Typical Meteorological Year (TMY) data sets hold hourly values of solar radiation and meteorological elements for a 1-year period. They are intended to be used for computer simulations of solar energy conversion systems and building systems for performance comparisons of different system types, configurations, and locations in the United States and its territories. Wilcox and Marion [13] go on to describe a TMY data set as being composed of 12 typical meteorological months (January through December) that are concatenated essentially without modification to form a single year with a serially complete data record for primary measurements. The monthly data sets contain actual time-series meteorological measurements and modeled solar values, though some hourly values may contain filled or interpolated data for periods when original observations are missing from the data archive.

\subsection{GHI modeling}

Salameh et al. [14] fit hourly solar irradiance data to Weibull, lognormal, and beta PDFs in order to describe the randomness of solar radiation. They used chi-squared and Kolmogorov-Smirnov testing to show that for a majority of the hourly data groups, a beta distribution function yielded the best goodness of fit results. Solar irradiance was also assumed to follow a beta distribution to provide the available solar 
power in source in Karaki et al. [15]. Therefore this study used beta PDFs to simulate GHI. The GHI beta distribution parameters were calculated from the closest TMY3 dataset, Fort Leonard Wood Army Air Field (AAF) (TMY 724457) to the subject microgrid locations. A TMY3 dataset consists of 8,760 lines which correspond to the 8,760 hours in a year beginning at $1 \mathrm{AM}$ on January $1^{\text {st }}$ and ending on $12 \mathrm{AM}$ on December $31^{\text {st }}$. For each dataset line, the GHI value is the total amount of direct and diffuse solar radiation received on a horizontal surface during the corresponding 60-minute time period (Wilcox and Marion, 2008). Therefore GHI values for TMY3 dataset lines that include sunrise or sunset are for a fraction of an hour and are not appropriate for use in the Guggenberger et al. (2012) model which requires hourly values.

The TMY3 Extraterrestrial Radiation Normal (ETRN) data were used to determine if the first and last value of GHI recorded for each day was representative of a full hour of sunlight. Wilcox and Marion (2008) define ETRN as the amount of solar radiation in $\mathrm{Wh} / \mathrm{m}^{2}$ received on a surface normal to the sun at the top of the atmosphere, and those values are typically on the order of $1,400 \mathrm{Wh} / \mathrm{m}^{2}$ for a full hour of sunlight for the subject TMY3 station. When the first and/or last ETRN values for a day were lower than the other values for that day, the first and/or last GHI measurements for that day were not retained.

The GHI values were normalized for the PV array geometry angle of incidence and corresponding pointing error. Pointing error was determined by first calculating the solar altitude angle for each hour using the equation given by Masters [16] using location latitude $(L)$, solar declination $(\delta)$, solar hour angle $(H)$, and the day number $(n)$ :

$$
\beta=\sin (L) \sin (\delta)+\cos (L) \cos (\delta) \cos (H)
$$

where

$$
\delta=23.45 \sin \left[\frac{360}{365}(n-81) \operatorname{deg}\right]
$$

Pointing error was calculated using equation (3). The GHI values were then normalized by dividing each retained hourly value by its corresponding pointing error. The normalized GHI values were then separated by month, and then grouped according to hour.

$$
\text { pointing error }=\sin (\beta)
$$

Ang and Tang [17] state that the beta distribution is appropriate for a describing random variable whose values are bounded between a maximum and minimum using the two shape parameters $(P$ and $Q)$ and two parameters representing the minimum $(A)$ and the maximum $(B)$. Those authors give the beta $\mathrm{PDF}$ as a function of time $(t)$ as:

$$
f(t)=\frac{1}{B(P, Q)} \frac{(t-A)^{(P-1)}(B-t)^{(Q-1)}}{(B-A)^{(P+Q-1)}}, P>0, Q>0, A<t<B
$$

where

$$
B(P, Q)=\frac{\Gamma(P) \Gamma(Q)}{\Gamma(P+Q)}
$$

All of the normalized GHI values for each full hour of sunlight for every month were assembled into a single dataset, and A, B, P, and Q were calculated for each using NCSS Statistical Software 9.0.15. Table 1 lists the beta distribution parameters $\mathrm{P}$ and $\mathrm{Q}$ for each hour every month. Strong positive correlations from hour to hour in GHI were found using Minitab® 16. Correlations showed a high probability of a large GHI value occurring in a given hour if the previous hour also yielded a large GHI value. Correlation values ranged from 23 percent to 94 percent with a mean of 77 percent. These correlations were included in the model. 
Table 1. P and Q beta parameters

\begin{tabular}{|c|c|c|c|c|c|c|c|c|c|c|c|c|c|}
\hline \multirow{2}{*}{\multicolumn{2}{|c|}{ Month Parameter }} & \multicolumn{12}{|c|}{ Hour } \\
\hline & & 7 & 8 & 9 & 10 & 11 & 12 & 13 & 14 & 15 & 16 & 17 & 18 \\
\hline \multirow{2}{*}{ Jan } & $P$ & & & & 1.2 & 0.71 & 1.3 & 0.79 & 4.7 & 2.2 & & & \\
\hline & $Q$ & & & & 1.0 & 0.31 & 1.6 & 1.2 & 11 & 2.3 & & & \\
\hline \multirow{2}{*}{ Feb } & $P$ & & & & 1.8 & 1.4 & 2.0 & 2.9 & 2.9 & 5.3 & & & \\
\hline & $Q$ & & & & 1.9 & 1.0 & 1.5 & 2.8 & 2.2 & 4.8 & & & \\
\hline \multirow{2}{*}{ Mar } & $P$ & & & 0.86 & 0.59 & 0.98 & 0.92 & 1.7 & 1.0 & 1.3 & & & \\
\hline & $Q$ & & & 1.4 & 0.46 & 1.5 & 1.9 & 3.3 & 1.6 & 2.3 & & & \\
\hline \multirow{2}{*}{ Apr } & $P$ & & 1.5 & 1.2 & 1.4 & 2.2 & 1.7 & 2.3 & 3.2 & 2.8 & 3.0 & 3.0 & \\
\hline & $\mathrm{Q}$ & & 2.5 & 1.3 & 1.4 & 2.5 & 1.5 & 1.9 & 3.4 & 2.2 & 3.3 & 2.8 & \\
\hline \multirow{2}{*}{ May } & $P$ & & 4.6 & 3.1 & 1.2 & 1.6 & 4.9 & 2.1 & 3.5 & 2.7 & 2.4 & 1.4 & 1.5 \\
\hline & $\mathrm{Q}$ & & 5.5 & 0.75 & 0.3 & 1.1 & 3.6 & 1.4 & 1.1 & 1.1 & 0.6 & 0.6 & 1.1 \\
\hline \multirow{2}{*}{ Jun } & $P$ & 4.9 & 3.9 & 3.3 & 2.2 & 1.6 & 1.5 & 1.9 & 2.0 & 2.5 & 1.9 & 1.6 & 3.0 \\
\hline & $\mathrm{Q}$ & 7.6 & 3.2 & 1.5 & 0.95 & 0.83 & 0.86 & 0.89 & 1.4 & 1.9 & 1.1 & 0.79 & 2.0 \\
\hline \multirow{2}{*}{ July } & $P$ & & 8.2 & 7.3 & 5.7 & 5.2 & 2.1 & 2.5 & 3.7 & 1.6 & 1.7 & 2.8 & 3.9 \\
\hline & $\mathrm{Q}$ & & 5.9 & 2.5 & 0.89 & 0.83 & 0.5 & 0.8 & 1.2 & 0.78 & 0.4 & 0.63 & 0.61 \\
\hline \multirow{2}{*}{ Aug } & $P$ & & 3.5 & 2.6 & 1.4 & 1.5 & 2.0 & 2.1 & 4.3 & 4.3 & 2.2 & 4.7 & \\
\hline & $\mathrm{Q}$ & & 4.8 & 1.8 & 0.53 & 0.59 & 1.4 & 1.3 & 2.0 & 2.4 & 0.87 & 1.4 & \\
\hline \multirow{2}{*}{ Sep } & $P$ & & & 1.8 & 1.3 & 1.1 & 3.3 & 1.6 & 3.5 & 1.3 & 1.8 & 2.2 & \\
\hline & $\mathrm{Q}$ & & & 1.2 & 0.65 & 0.86 & 3.9 & 1.2 & 3.7 & 0.92 & 1.2 & 1.8 & \\
\hline \multirow{2}{*}{ Oct } & $P$ & & & 1.1 & 0.95 & 0.90 & 0.93 & 0.94 & 0.82 & 1.3 & 2.4 & & \\
\hline & $\mathrm{Q}$ & & & 0.85 & 0.50 & 0.54 & 0.66 & 0.52 & 0.54 & 1.0 & 1.1 & & \\
\hline \multirow{2}{*}{ Nov } & $P$ & & & & 1.2 & 1.7 & 1.5 & 1.9 & 1.4 & 1.4 & 1.7 & & \\
\hline & $\mathrm{Q}$ & & & & 1.6 & 2.4 & 1.9 & 2.6 & 1.5 & 1.5 & 1.8 & & \\
\hline \multirow{2}{*}{ Dec } & $P$ & & & & 1.5 & 1.3 & 1.5 & 1.5 & 1.7 & 1.9 & 2.5 & & \\
\hline & $\mathrm{Q}$ & & & & 1.8 & 1.1 & 1.4 & 1.4 & 1.5 & 1.6 & 1.9 & & \\
\hline
\end{tabular}

\subsection{Ambient temperature modeling}

Ambient temperature $\left({ }^{\circ} \mathrm{C}\right)$ was modeled from TMY3 data for Fort Leonard Wood based on the time of day for each month. For each day, data was broken down into three categories: morning cooling, warming, and evening cooling. The cooling period was separated into two categories due to different trends in the data that are shown in the Fig. 2 example.

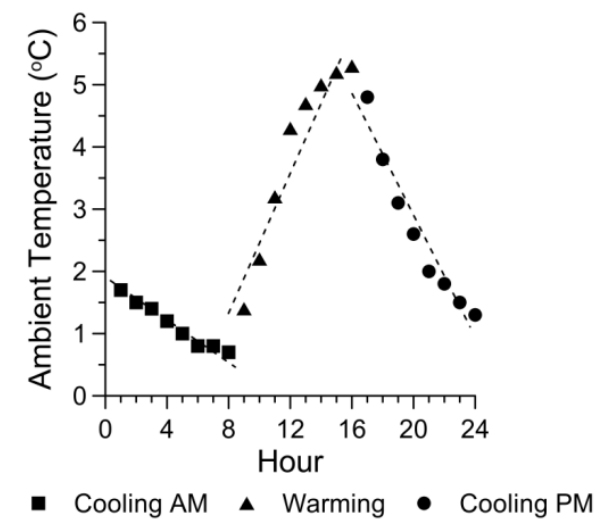

Fig. 2. Ambient temperature trends for January.

Ambient temperature trends were created for all 12 months. A probability plot analysis showed that the data fit a lognormal PDF where the conditional shape was given by the equation of the trend line. These equations and the corresponding shape and scale factors are listed in Table 2. Strong positive correlations from hour to hour in ambient temperature were also found using Minitab® 16. Correlations showed a high probability of a high temperature occurring in a given hour if the previous hour also had a high temperature, and the same with low temperatures. Correlation values ranged from 73 percent to 100 percent with an average of 97 percent. These correlations were included in the model. 
Table 2. Ambient temperature trend line equations

\begin{tabular}{cccc}
\hline Month & $\begin{array}{c}\text { Cooling AM } \\
\left(y\left({ }^{\circ} \mathrm{C}\right)=\right)\end{array}$ & $\begin{array}{c}\text { Warming } \\
\left(y\left({ }^{\circ} \mathrm{C}\right)=\right)\end{array}$ & $\begin{array}{c}\text { Cooling PM } \\
\left(y\left({ }^{\circ} \mathrm{C}\right)=\right)\end{array}$ \\
\hline January & $-0.149 x+1.81$ & $0.573 x+1.34$ & $-0.482 x+4.782$ \\
February & $-0.271 x+2.75$ & $0.968 x+3.21$ & $-0.739 x+8.34$ \\
March & $-0.148 x+4.34$ & $0.498 x+5.54$ & $-0.441 x+8.68$ \\
April & $-0.214 x+8.89$ & $0.956 x+9.26$ & $-1.01 x+16.6$ \\
May & $-0.500 x+15.5$ & $1.33 x+15.7$ & $-1.17 x+25.0$ \\
June & $-0.387 x+22.1$ & $1.04 x+21.9$ & $-0.907 x+29.4$ \\
July & $-0.529 x+24.2$ & $1.34 x+23.3$ & $-1.08 x+32.5$ \\
August & $-0.250 x+21.0$ & $0.941 x+22.8$ & $-0.941 x+28.7$ \\
September & $-0.339 x+16.8$ & $1.05 x+17.1$ & $-0.847 x+23.5$ \\
October & $-0.214 x+13.9$ & $1.16 x+14.8$ & $-1.05 x+21.4$ \\
November & $-0.195 x+3.60$ & $0.466 x+3.49$ & $-0.323 x+6.09$ \\
December & $-0.194 x+1.46$ & $0.558 x+1.60$ & $-0.411 x+4.46$ \\
\hline
\end{tabular}

\subsection{VRB SOC modeling}

Nguyen et al. [12] described VRBs as a flow-type battery that stores chemical energy and produces electricity using reduction-oxidation (redox) reactions between vanadium in the electrolytes. The batteries include two closed electrolyte circuits where, in each circuit, the electrolyte is stored in a separate tank and circulated though the cell stacks where the electrochemical reactions take place. Nguyen et al. [12] also state that charge and discharge operations of a VRB are dependent on the SOC, the energy load, and the power produced by the PV array. During the discharge stage of operations, the VRB supplies power to the energy load, and pumps within the VRB. During the charging stage of operation, the pumps within the VRB cycle the electrolytes stored in the tanks through the cell stacks. VRB capacity changes due to the amount of energy going in and out of the system at any given time. Therefore VRB SOC can be determined from the capacity using the Guggenberger et al. [6] equation:

$$
\text { VRB SOC }=\left[\frac{9}{20}\left(\operatorname{VRB} \text { Capacity }+\frac{10}{9}\right)\right]
$$

The VRB energy capacity for the system at TA-246 was a maximum of $20 \mathrm{kWh}$. The VRB initial SOC was assumed to fit a uniform PDF. The lower threshold of the PDF was assumed to be 30 percent of the overall maximum charge, while the upper threshold for the distribution was chosen to be 80 percent of the overall maximum charge. The lower threshold was chosen because at a charge of 30 percent, the system switches from running off of the VRB to running on the generator. The upper threshold of the VRB charge capacity was chosen because once the VRB is re-charged to at least 80 percent, the system then switches back to running off the VRB [6]. The uniform distribution for the VRB SOC has a minimum value of $6 \mathrm{kWh}$ and a maximum value of $20 \mathrm{kWh}$.

The B2222 microgrid used similar SOC thresholds except that the lower threshold was assumed to be 20 percent of the overall maximum charge and the upper threshold was assumed to be 73 percent in order to be consistent with the system described in Nguyen et al. [12]. The uniform distribution for the VRB $\mathrm{SOC}$ for this system has a minimum value of $4 \mathrm{kWh}$ and a maximum value of $14.6 \mathrm{kWh}$.

\subsection{Energy load modeling}

Eight months of energy load data were available from the B2222 office building, and those data were used to develop the load PDF. An example of the energy load over a one-month period is shown in Fig. 3. The collected monthly energy load data generally ranged between $480-1,500 \mathrm{kWh}$, with higher energy load demands occurring in the summer months. The load data were compiled into hourly groups ranging from $1 \mathrm{AM}$ to $12 \mathrm{AM}$, each hourly data set fit a lognormal distribution.Parameters determined from the lognormal probability plots were used to generate PDFs to predict the values of energy load at any given hour. 


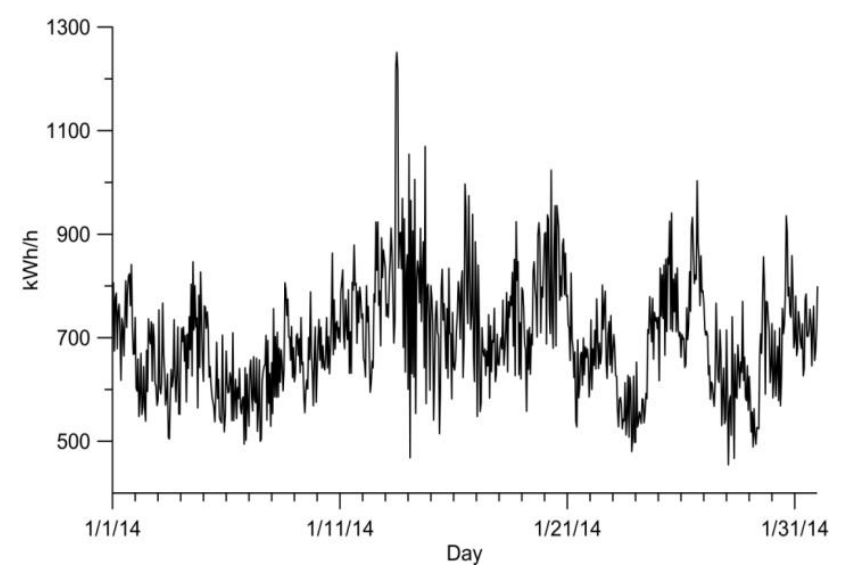

Fig. 3. Hourly energy load profile for January 2014.

\subsection{Modeling process}

Microgrids TA-246 and B2222 were modeled to operate in either renewable mode or in diesel mode based on the modeled VRB SOC.

1. Renewable mode. This mode operated when the energy load was powered by the VRB and the available PV systems power. The microgrid was renewable mode when the VRB SOC greater than the lower threshold SOC, or when the PV power was sufficient to both charge the VRB and supply power to energy load.

2. Diesel mode. When the VRB was in discharging stage and the SOC was lower than the lower threshold SOC, the system switched to diesel mode. This mode operated when the energy load was powered by the diesel self-regulated generator as well as the PV array. The system operated in this mode until the VRB SOC was greater than the upper threshold SOC.

The Guggenberger et al. [6] model predicted the microgrid performance according to equation (6),

$$
P_{\text {Out MPPT }}+P_{V R B}+P_{D C \text { Load }}=h_{\text {inverter }}\left(P_{H V A C}+P_{A C \text { Load }}+P_{\text {Generator }}\right)
$$

where $P_{\text {Out } M P P T}$ is the power available to the system after the maximum power point tracking (MPPT), $P_{V R B}$ is the power charged or discharged from the VRB, $P_{D C}$ Load is the peak DC load available to the system, $P_{H V A C}$ is the power used by the VRB's HVAC system, $P_{A C \text { Load }}$ is the peak AC load that is available to the system, $P_{\text {Generator }}$ is the power of the generator, and $h_{\text {inverter }}$ is the efficiency of the inverter.

Monte Carlo modeling of the microgrids at TA-246 and B2222 was performed using the Oracle ${ }^{\circledR}$ Crystal Ball Version 11.1.275.0 spreadsheet-based application for Microsoft Excel.

1. Method I. GHI, ambient temperature, VRB SOC, and the energy load were all modeled as random variables. For these variables, PDFs were used to generate values based on a probability distribution fit to a data set for each trial the model runs. One initial VRB SOC was simulated for January $1^{\text {st }}$ at 1 AM. This scenario modeled continuous operation of the microgrid from January through December.

2. Method II. This scenario was identical to Method I except that a unique VRB SOC was stochastically generated for the first hour of each month. This scenario modeled discontinuous operation of the microgrid on a monthly interval.

3. Method III. This scenario was identical to Method I except that it was assumed that the VRB was recharged at the beginning of each month, and the VRB SOC was set to $16 \mathrm{kWh}$ for TA-246 and $14.6 \mathrm{kWh}$ for B2222 for the first hour of each month.

A sensitivity analysis was performed on the number of Monte Carlo simulations as a quality control measure, and the output ensemble of the diesel generator operation time was found to stabilize at 1,000 realizations. 


\section{Results}

The percent of time the generator was running the system is recorded for each month of the year. The average percent time the generator was running is calculated for each month and compared to the results of the other two methods. The results of all three methods are also compared to the deterministic model used in Guggenberger et al. [6]. Stochastic results for TA-246 show that the average annual operating times for the generator in Methods I, II, and III were relatively close. The average percent of the generator running time was 14.96 percent for Method I, 15.08 percent for Method II, and 14.96 percent for Method III. These stochastic predations agreed with the deterministic prediction of 14.8 percent for the year. Fig. 4 shows that there are more significant differences at monthly intervals although the stochastic results were relatively similar. Differences in the modeling results from the deterministic results in the later months due to having partial energy load sets.

The stochastic results for the B2222 microgrid were also compared to the deterministic modeling of that system. Methods I, II, and III all still showed performance results that were similar to one another, however, they did differ from the deterministic results. Method I, II, and III results for the months of June, July, and August were the same as the results of the deterministic model. For the year, the percent time generator running for Method I was 13.47 percent, Method II was 13.54 percent, and Method III was also 13.47 percent. The deterministic model time generator running averaged 13.70 percent for the year.

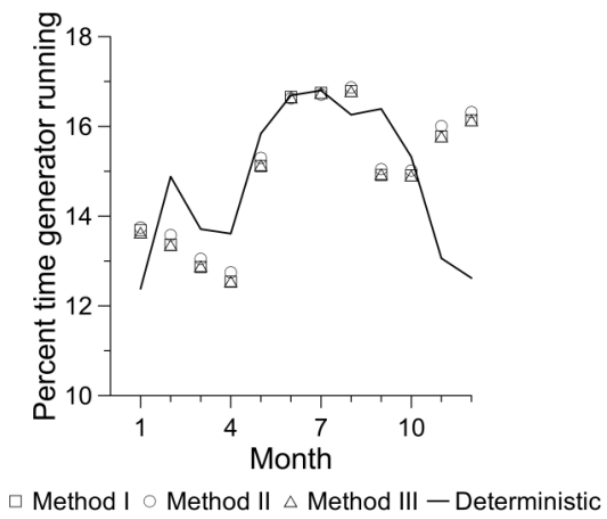

(a)

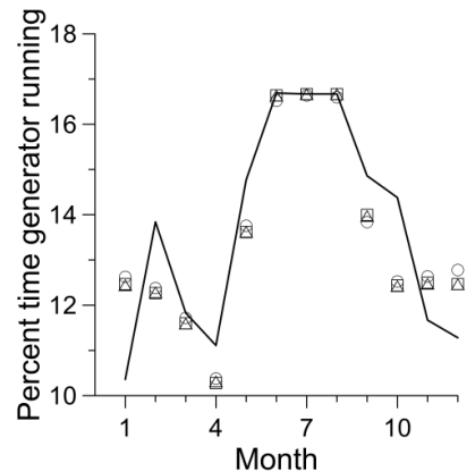

$\square$ Method I $\circ$ Method II $\triangle$ Method III - Deterministic

(b)

Fig. 4. Stochastic results compared to deterministic method: (a) TA-246 and (b) B2222.

Altering the VRB SOC in three methods also shows that changing the way VRB SOC is treated in the model does have a slight effect on the predicted performance, but does not make a significant change. This is due to the VRB SOC only being altered for one hour at the beginning of each year/month with the following SOCs being calculated from equation (5). The VRB is also constantly being recharged throughout each day in order to keep the SOC within the upper and lower thresholds.

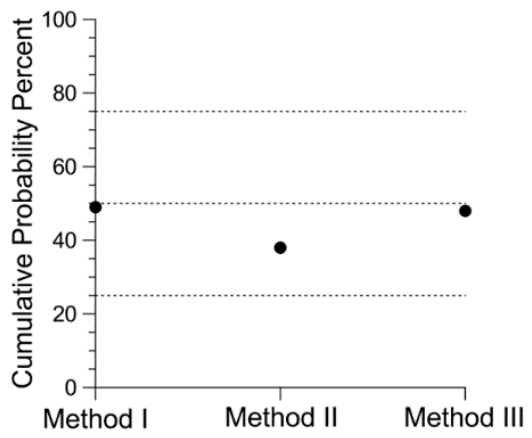

Fig. 5. Comparison of B2222 modeling results to measured performance. 
The model results of B2222 are also compared to real performance data collected from the B2222 site from May 2013 and shown in Fig. 5. The observed number of hours that the system operated on the grid was compared to the simulated cumulative percent of the time the generator was running. For May 2013, B2222 operated on the grid for 102 hours. For Method I, the generator shows a 49 percent probability of running at least 102 hours or less. For Method II, the generator has a 38 percent probability or less of running for that amount of time or less, and for Method III, the generator has a 48 percent probability. All three values were in the central range of the cumulative probability range of the 25 to 75 percent. Additional months of performance data were not available to assess if the stochastic models would tend to over or under predict the use of the diesel generator. The deterministic results predicted that the system would run the diesel generator for a total of 107 hours for the month of May.

\section{Conclusion}

This study stochastically characterized GHI and ambient temperature using a TMY3 data set, VRB SOC was characterized using engineering judgment, and energy load were stochastically characterized using observed data. PDFs of the random variables were incorporated into a deterministic PV performance model in order to create a Monte Carlo based stochastic PV performance model. The analysis for three different initial SOC scenarios indicated that the stochastic analysis was relatively insensitive to the initial state of the battery. Finally, there were insufficient microgrid performance data available to conclusively characterize the reliability of the stochastic model. However, the available data indicated that the modeling techniques presented in this paper may have the potential for wider applicability given the nationwide availability of TMY3 data. The applicability could be expanded further by modifying the basic model to include other energy systems beyond VRBs.

\section{References}

[1] Provata E, Kolokotsa D, Papantoniou S, Pietrini M, Giovannelli A, Romiti G. Delvelopment of optimization algorithms for the leaf community microgrid. Renewable Energy, 2015; 74(2015):782-795.

[2] Patterson M, Macia NF, Kannan AM. Hybrid microgrid model based on solar photovoltaic battery fuel cell system for intermittent load applications. IEEE Trans. Energy Convers, 2015; 30(1):359-366.

[3] Merei G, Berger C, Sauer DU. Optimization of an off-grid hybrid PV-wind-diesel system with different battery technologies using genetic algorithm. Solar Energy, 2013; 97(2013):460-473.

[4] Bandara K, Sweet T, Ekanayake J. Photovoltaic applications for off-grid electrification using novel multi-level inverter technology with energy storage. Renewable Energy, 2012; 37(2012):82-88.

[5] Fossati JP, Galarza A, Martin-Villate A, Fontan L. A method for optimal sizing energy storage systems for microgrids. Renewable Energy, 2015; 77(2015):539-549.

[6] Guggenberger JD, Elmore AC, Tichenor JL, Crow ML. Performance prediction of a vanadium redox battery for use in portable, scalable microgrids. IEEE Transactions on Smart Grid, 2012; 3(4):2109-2116.

[7] Alenezi FQ, Sykulski JK, Rotaru M. Grid-connected photovoltaic module and array sizing based on an iterative approach. International Journal of Smart Grid and Clean Energy, 2013; 3(2):247-254.

[8] Huang CJ, Huang MT, Chen CC. A novel power output model for photovoltaic systems. International Journal of Smart Grid and Clean Energy, 2013; 2(2):139-147.

[9] Arabali A, Ghofani M, Etezadi-Amoli M, Fadali MS. Stochastic performance assessment and sizing for a hybrid power systems of solar/wind/energy storage. IEEE Trans. Sustainable Energy, 2014; 5(2):363-371.

[10] Kishore LN, Fernandez E. Reliability well-being assessment of PV-wind hybrid system using Monte Carlo simulation. In: Proc. Int. Conf. Emerging Trends in Electrical and Computer Technology, 2011:63-68.

[11] Khatod DK, Pant V, Sharma J. Analytical approach for well-being assessment of small autonomous power systems with solar and wind energy sources. IEEE Trans. Energy Convers., 2010; 25(2):535-545.

[12] Nguyen TA, Xin Q, Guggenberger JD, Crow ML, Elmore AC. Performance characterization for photovoltaic-vanadium redox battery microgrid systems. IEEE Trans. on Sustainable Energy, 2014; 5(4):1379-1388.

[13] Wilcox S, Marion W. User's Manual for TMY3 Data Sets. Golden, CO: Renewable Energy Laboratory (NREL); 2008.

[14] Salameh ZM, Borowy BS, Amin ARA. Photovoltaic module-site matching based on capacity factors. IEEE Transactions on Energy Convers., 1995; 10(2):326-331. 
[15] Karaki SH, Chedid RB, Ramadan R. Probabilistic performance assessment of autonomous solar-wind energy conversion systems. IEEE Trans. on Energy Convers., 1999; 14(3):766-772.

[16] Masters GM. Renewable and Efficient Electrical Power Systems. 2nd ed. Hoboken, NJ: John Wiley \& Sons, Inc.; 2013.

[17] Ang AHS, Tang WH. Probability Concepts in Engineering Planning and Design Volume I - Basic Principles. 1st ed. New York: John Wiley \& Sons, Inc.; 1975. 